Few-Body Systems manuscript No.

(will be inserted by the editor)

Jose Manuel Alarcón • Astrid N. Hiller Blin •

Christian Weiss

\title{
Transverse densities of octet baryons from chiral effective field theory
}

\begin{abstract}
Transverse densities describe the distribution of charge and current at fixed light-front time and provide a frame-independent spatial representation of hadrons as relativistic systems. We calculate the transverse densities of the octet baryons at peripheral distances $b=O\left(M_{\pi}^{-1}\right)$ in an approach combining chiral effective field theory $(\chi \mathrm{EFT})$ and dispersion analysis. The densities are represented as dispersive integrals of the imaginary parts of the baryon electromagnetic form factors in the timelike region (spectral functions). The spectral functions on the two-pion cut at $t>4 M_{\pi}^{2}$ are computed using relativistic $\chi \mathrm{EFT}$ with octet and decuplet baryons in the EOMS renormalization scheme. The calculations are extended into the $\rho$-meson mass region, using a dispersive method that incorporates the timelike pion form-factor data. The approach allows us to construct densities at distances $b>1$ fm with controlled uncertainties. Our results provide insight into the peripheral structure of nucleons and hyperons and can be compared with empirical densities and lattice-QCD calculations.
\end{abstract}

Keywords Electromagnetic form factors $\cdot$ Chiral Lagrangians $\cdot$ Hyperons $\cdot$ Charge distribution

\section{Introduction}

Light-front quantization offers a natural framework for formulating the spatial structure of relativistic systems and exploring its connection to the underlying dynamics; see 1 for a review. In this framework, hadronic current matrix elements (vector, axial) are represented in terms of transverse densities, which are two-dimensional Fourier transforms of the invariant form factors and describe the transverse spatial distribution of charge and current in the hadron at fixed light-front time $x^{+} \equiv x^{0}+x^{3}, 2,3,4,5$, The transverse densities are frame independent (they are invariant under longitudinal boosts and transform kinematically under transverse boosts) and thus provide an objective spatial representation of the hadron as a relativistic system. In composite models of hadron structure they correspond to proper densities of the light-front wave functions of the system. In the context of QCD the transverse charge and current densities in hadrons can be related to the generalized parton distributions (GPDs) describing the distribution of quarks and antiquarks in longitudinal momentum and transverse position [3; 6]. The charge and magnetization densities in the nucleon have been extracted from the available electromagnetic form-factor data 7 , 8 and provide interesting insight into the nucleon structure; see [5] for a review. It is worthwhile to explore how the transverse densities could be calculated using theoretical methods, and how the studies could be extended to other baryons.

Jose Manuel Alarcón and Christian Weiss

Theory Center, Jefferson Lab, Newport News, VA 23606, USA

E-mail: alarcon@jlab.org, E-mail: weiss@jlab.org

Astrid N. Hiller Blin

Departamento de Física Teórica and IFIC, Centro Mixto Universidad de Valencia-CSIC, Institutos de Investigación de Paterna, E-46071 Valencia, Spain

E-mail: astrid.hiller@uv.es 
The transverse densities at a given distance can be connected with the "exchange mechanisms" acting in the hadron form factors - virtual processes in which the current couples to the hadron through the exchange of a hadronic system in the $t$-channel. This connection can be made rigorous in a dispersive representation of the transverse densities [9, 10. At distances $b=O\left(M_{\pi}^{-1}\right)$, the densities are governed by soft-pion exchange between the current and the hadron and can be calculated modelindependently using chiral effective field theory $(\chi \mathrm{EFT})$. Detailed studies of the peripheral densities in the nucleon were performed in [10, 11; 12 using $\chi \mathrm{EFT}$ with $\mathrm{SU}(2)$ flavor group, and a simple quantummechanical interpretation of the results was obtained (chiral light-front wave functions, orbital motion of a peripheral pion). At distances $b \sim 1 \mathrm{fm}$, the transverse densities are dominated by vector-meson exchange (isovector $\rho$; isoscalar $\omega, \phi$ ) and offer interesting insight into the duality between quark structure and meson exchange 14.

In these proceedings we report about a study of the peripheral densities of the SU(3) flavoroctet baryons combining methods of $\chi \mathrm{EFT}$ and dispersion analysis. The densities are represented as a dispersive integral over the imaginary parts of the baryon electromagnetic form factors in the timelike region (spectral functions). The isovector spectral functions on the two-pion cut at $t>4 M_{\pi}^{2}$ are computed using relativistic $\chi \mathrm{EFT}$ with octet and decuplet baryons in the EOMS renormalization scheme. The calculations are extended into the $\rho$-meson mass region using a dispersive method that incorporates the timelike pion form-factor data. The methods allow us to construct the densities down to distances $b \sim 1 \mathrm{fm}$ with controlled uncertainties. Details will be reported in a forthcoming publication 13 .

\section{Transverse densities and dispersive representation}

The matrix element of the electromagnetic current between states of a flavor-octet baryon $(B=$ $N, \Sigma, \Xi)$ with 4-momenta $p$ and $p^{\prime}$ is described by two invariant form factors, $F_{1}^{B}(t)$ and $F_{2}^{B}(t)$ (the Dirac and Pauli form factors; we follow the conventions of [10]). They are functions of the invariant momentum transfer $t=\Delta^{2}=\left(p^{\prime}-p\right)^{2}$, and can be measured and interpreted without specifying the form of relativistic dynamics or the reference frame. In the context of light-front quantization, one considers the current matrix element in a frame where the 4-momentum transfer has only transverse components $\Delta^{+}=\Delta^{-}=0, \Delta_{T} \neq 0$, and represents the form factors as Fourier transforms of certain two-dimensional densities (here $b \equiv|\mathbf{b}|$ ),

$$
F_{i}^{B}\left(t=-\left|\boldsymbol{\Delta}_{T}\right|^{2}\right)=\int \mathrm{d}^{2} b e^{i \boldsymbol{\Delta}_{T} \cdot \mathbf{b}} \rho_{i}^{B}(b) \quad(i=1,2) .
$$

The interpretation of $\rho_{1,2}(b)$ as spatial densities is discussed in [3, 5] and summarized in [10]. Their spatial integral reproduces the total charge and anomalous magnetic moment of the baryon. In a state where the baryon is localized in transverse space at the origin, $\rho_{1}^{B}(b)$ describes the spin-independent $J^{+}$current at light-front time $x^{+}=0$ and transverse position $\mathbf{b}$, while $\widetilde{\rho}_{2}^{B}(b) \equiv \partial / \partial b\left[\rho_{2}^{B}(b) /\left(2 M_{B}\right)\right]$ describes the spin-dependent part of the current in a transversely polarized nucleon.

The form factors are analytic functions of $t$ and satisfy unsubtracted dispersion relations

$$
F_{i}^{B}(t)=\int_{4 M_{\pi}^{2}}^{\infty} \frac{\mathrm{d} t^{\prime}}{t^{\prime}-t-i 0} \frac{\operatorname{Im} F_{i}^{B}\left(t^{\prime}\right)}{\pi} \quad(i=1,2) .
$$

The spectral functions are given by the imaginary parts of the form factors on the principal cut starting at $t=4 M_{\pi}^{2}$. They describe processes in which the current at timelike $t>0$ converts to a hadronic state that couples to the $B \bar{B}$ system. Most of the relevant processes are in the unphysical region below the two-baryon threshold at $t=4 M_{B}^{2}$, where the spectral function can only be computed theoretically. From Eqs. (1) and (2), one obtains a dispersive representation of the transverse densities [9]

$$
\begin{aligned}
& \rho_{1}^{B}(b)=\int_{4 M_{\pi}^{2}}^{\infty} \mathrm{d} t \frac{K_{0}(\sqrt{t} b)}{2 \pi} \frac{\operatorname{Im} F_{1}^{B}(t)}{\pi}, \\
& \widetilde{\rho}_{2}^{B}(b)=-\int_{4 M_{\pi}^{2}}^{\infty} \mathrm{d} t \frac{\sqrt{t} K_{1}(\sqrt{t} b)}{4 \pi M_{B}} \frac{\operatorname{Im} F_{2}^{B}(t)}{\pi},
\end{aligned}
$$



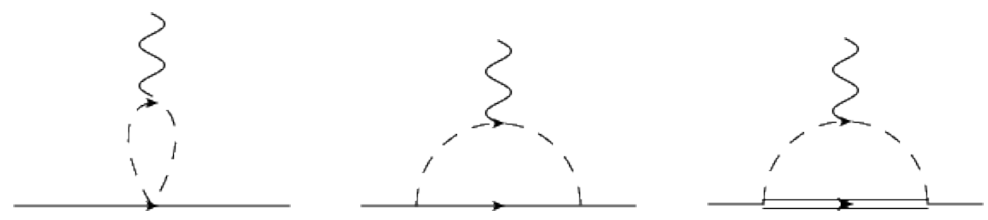

Fig. 1 Chiral processes contributing to the spectral functions of the baryon form factors $\operatorname{Im} F_{1,2}^{B}(t)$ at $\mathcal{O}\left(p^{3}\right)$. Dashed lines: pions/kaons. Solid lines: octet baryons. Double lines: decuplet baryons.

where $K_{n}(n=0,1)$ are the modified Bessel functions. The integrals in Eqs. (3) and (4) converge exponentially at large $t, K_{n}(\sqrt{t} b) \sim \exp (-\sqrt{t} b)$ at $\sqrt{t} b \gg 1$, strongly suppressing contributions from high-mass hadronic states. Depending on the distance $b$, the integrals sample the spectral functions in different regions of $t$ [14. At $b>2 \mathrm{fm}$, the integrals extend over the near-threshold region $t=$ $4 M_{\pi}^{2}+$ few $M_{\pi}^{2}$, where the spectral functions arise from soft two-pion exchange between the baryon and the current. At $b \sim 1 \mathrm{fm}$, the dominant contributions come from the vector-meson region of the spectral functions $t \sim M_{V}^{2}\left(V=\rho^{0}, \omega, \phi\right)$. At even shorter distances, the integrals extend over the high-mass region $t>1 \mathrm{GeV}^{2}$, where the spectral functions involve multi-hadron states and are poorly known at present [15. The dispersive representation Eqs. (3) and (4) thus establishes a quantitative connection between the transverse densities and the exchange mechanisms in the form factor.

\section{Spectral functions from chiral EFT and dispersion theory}

We compute the isovector spectral functions of the SU(3) octet-baryon form factors above the twopion threshold using relativistic $\chi$ EFT. Decuplet baryons are included as dynamical degrees of freedom within the small-scale expansion (SSE) 16; 17] (the first form factor calculations in this approach were performed in [18]). The fields and Lagrangian are described in [19, 20, 21]. The renormalization of the divergent pieces is performed within the EOMS scheme, which permits consistent power counting 22 . The spectral functions arise from diagrams with a two-pion cut; the $O\left(p^{3}\right)$ diagrams are shown in Fig. 1 (the imaginary parts are actually finite at this order and do not require renormalization). The low-energy constants at this order are given by the nucleon's axial charge (or $\pi N N$ coupling) and the $\pi N \Delta$ coupling, so that the spectral functions are $\chi \mathrm{EFT}$ predictions free of unknown parameters. While not shown here, we have calculated the entire form factor from the full set of $\mathcal{O}\left(p^{3}\right)$ diagrams in order to verify gauge invariance, and reproduce the $\mathrm{SU}(2)$ results of $[21$.

The $\chi$ EFT expressions by themselves describe the baryon spectral functions only in the nearthreshold region $t=4 M_{\pi}^{2}+$ few $M_{\pi}^{2}$. In order to extend the description to higher $t$, we use a dispersive technique following [23, 24, 25]; see also [26]. On general grounds, the isovector spectral function on the two-pion cut (neglecting the contributions from $4 \pi$ states) can be expressed as

$$
\operatorname{Im} F_{i}^{B}(t)=\frac{k_{\mathrm{cm}}^{3}}{\sqrt{t}} \Gamma_{i}^{B}(t) F_{\pi}^{*}(t) \quad(i=1,2),
$$

where $k_{\mathrm{cm}}=\sqrt{t / 4-M_{\pi}^{2}}$ is the $t$-channel center-of-mass momentum, $\Gamma_{i}^{B}(t)$ is the $I=J=1 \pi \pi \rightarrow \bar{B} B$ partial-wave amplitude, and $F_{\pi}(t)$ is the pion form factor. The expression on the right-hand side of Eq. $(5)$ is real because the complex functions $\Gamma_{i}^{B}(t)$ and $F_{\pi}(t)$ have the same phase on the two-pion cut (Watson theorem). It is convenient to rewrite Eq. (5) as

$$
\operatorname{Im} F_{i}^{B}(t)=\frac{k_{\mathrm{cm}}^{3}}{\sqrt{t}} \frac{\Gamma_{i}^{B}(t)}{F_{\pi}(t)}\left|F_{\pi}(t)\right|^{2} \quad(i=1,2) .
$$

This representation has two major advantages: (i) The function $\Gamma_{i}^{B}(t) / F_{\pi}(t)$ has no two-pion cut, because it is real at $t>4 M_{\pi}^{2}$; (ii) the squared modulus $\left|F_{\pi}(t)\right|^{2}$ can be extracted directly from the $e^{+} e^{-} \rightarrow \pi^{+} \pi^{-}$exclusive annihilation cross section, without determining the phase of the complex function. We now use $\chi \mathrm{EFT}$ to calculate the real function $\Gamma_{i}^{B}(t) / F_{\pi}(t)$ at $t>4 M_{\pi}^{2}$, and multiply with the empirical $\left|F_{\pi}(t)\right|^{2}$ containing the $\rho$ meson resonance. At $O\left(p^{3}\right)$ one has $F_{\pi}(t) \equiv 1$, so that 


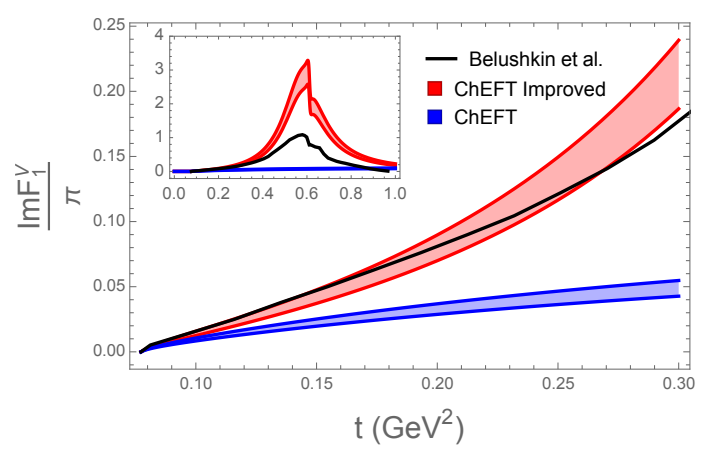

Fig. 2 Dispersive improvement of the $\chi$ EFT spectral function $\operatorname{Im} F_{1}(t)$, Eq. (7). The main figure shows the spectral functions up to $t=0.3 \mathrm{GeV}^{2}$, the inset figure shows them up to $1 \mathrm{GeV}^{2}$. Blue band: $\chi \mathrm{EFT}$ result at $O\left(p^{3}\right)$, including $N$ and $\Delta$ intermediate states. Red band: Improved spectral function Eq. (7). Black line: Dispersiontheoretical spectral function from [28].

the $\chi$ EFT result for the ratio $\Gamma_{i}^{B}(t) / F_{\pi}(t)$ is the same as that for the amplitude $\Gamma_{i}^{B}(t)$ itself, and the prescription simply amounts to multiplying the $O\left(p^{3}\right)$ result for the spectral function by $\left|F_{\pi}(t)\right|^{2}$,

$$
\operatorname{Im} F_{i}^{B}(t)[\text { improved }]=\operatorname{Im} F_{i}^{B}(t)\left[O\left(p^{3}\right)\right] \times\left|F_{\pi}(t)\right|^{2} \quad(i=1,2) .
$$

The prescription Eq. (7) results in a marked improvement of the $\chi$ EFT predictions for the spectral functions. The improved $\chi \mathrm{EFT}$ results for the nucleon $(B=N)$ reproduce the dispersion-theoretical spectral functions (obtained by analytic continuation of the $\pi N$ phase shifts $27,28,29$ ) up to $t \sim 16 M_{\pi}^{2}$ within errors, and have qualitatively the correct behavior even in the $\rho$-meson mass region (see Fig. 2). We use this method to calculate the other octet-baryon spectral functions on the two-pion cut. A detailed discussion of the procedure and its applications will be presented in [13.

\section{Peripheral transverse densities}

Using the improved $\chi \mathrm{EFT}$ results for the spectral functions, we calculate the peripheral isovector densities in the octet baryons. The restriction to distances $b>1 \mathrm{fm}$ ensures that the dispersion integrals Eqs. (3) and (4) extend only over the region of $t$ where our approximation to the spectral functions is justified. The uncertainties of the isovector densities are estimated by propagating the theoretical uncertainty of the spectral functions 13. In order to estimate also the isoscalar densities, we parametrize the isoscalar spectral functions of the octet baryons in the mass region $t<1 \mathrm{GeV}^{2}$ by vector-meson poles $(V=\omega, \phi)$. The $V B B$ couplings are obtained from $\mathrm{SU}(3)$ symmetry, with certain assumptions regarding the $F / D$ ratio and the empirical $V N N$ couplings (we do not aim for a precise description of the isoscalar sector here, as the peripheral densities are dominated by the isovector component).

Results for the transverse charge densities are shown in Fig. 3 . The densities decay exponentially at large $b$, as dictated by the analytic properties of Eqs. (3) and (4). The comparison of the various components reveals several interesting features. At large distances $b>3 \mathrm{fm}$, the densities are dominated by the isovector component, resulting from two-pion exchange near threshold. The isoscalar component generally becomes comparable to the isovector at distances below $b \sim 2 \mathrm{fm}$, due to the similar strength of the spectral functions in the vector-meson region ( $\rho$ vs. $\omega, \phi)$. In the neutron, the isovector component dominates for $b>1.5 \mathrm{fm}$, and causes the peripheral charge density to be negative. Studies of empirical neutron densities have shown that at $b \sim 1 \mathrm{fm}$ the isoscalar takes over, and the neutron charge density becomes positive [4; 14; at such distances the present theoretical calculation has large uncertainties and cannot predict the sign.

In the $\Lambda$ and $\Sigma^{0}$ charge densities, the isovector component is absent because of the isospin selection rules for the $t$-channel process current $\rightarrow B \bar{B}$ : the transitions $\left|I=1, I^{3}=0\right\rangle \rightarrow\left|I=0, I^{3}=0\right\rangle \mid I=$ $\left.0, I^{3}=0\right\rangle$ and $\left|I=1, I^{3}=0\right\rangle \rightarrow\left|I=1, I^{3}=0\right\rangle\left|I=1, I^{3}=0\right\rangle$ are both forbidden. In contrast, in the $\Lambda \Sigma^{0}$ transition density, the isoscalar component is absent and the density is pure isovector. The peripheral densities of the $\Lambda$ and $\Sigma^{0}$ thus provide a means to isolate the low-mass isovector and isoscalar exchanges in the form factors. The $\Xi^{0}$ and $\Xi^{-}$densities are similar to the proton and neutron in that both isovector and isoscalar exchanges are present. The isovector component is relatively smaller in the $\Xi$ states, due to the suppression of octet intermediate states in the pion loops for the $\Xi$ form factor (they are of the same order as the decuplet intermediate states). 

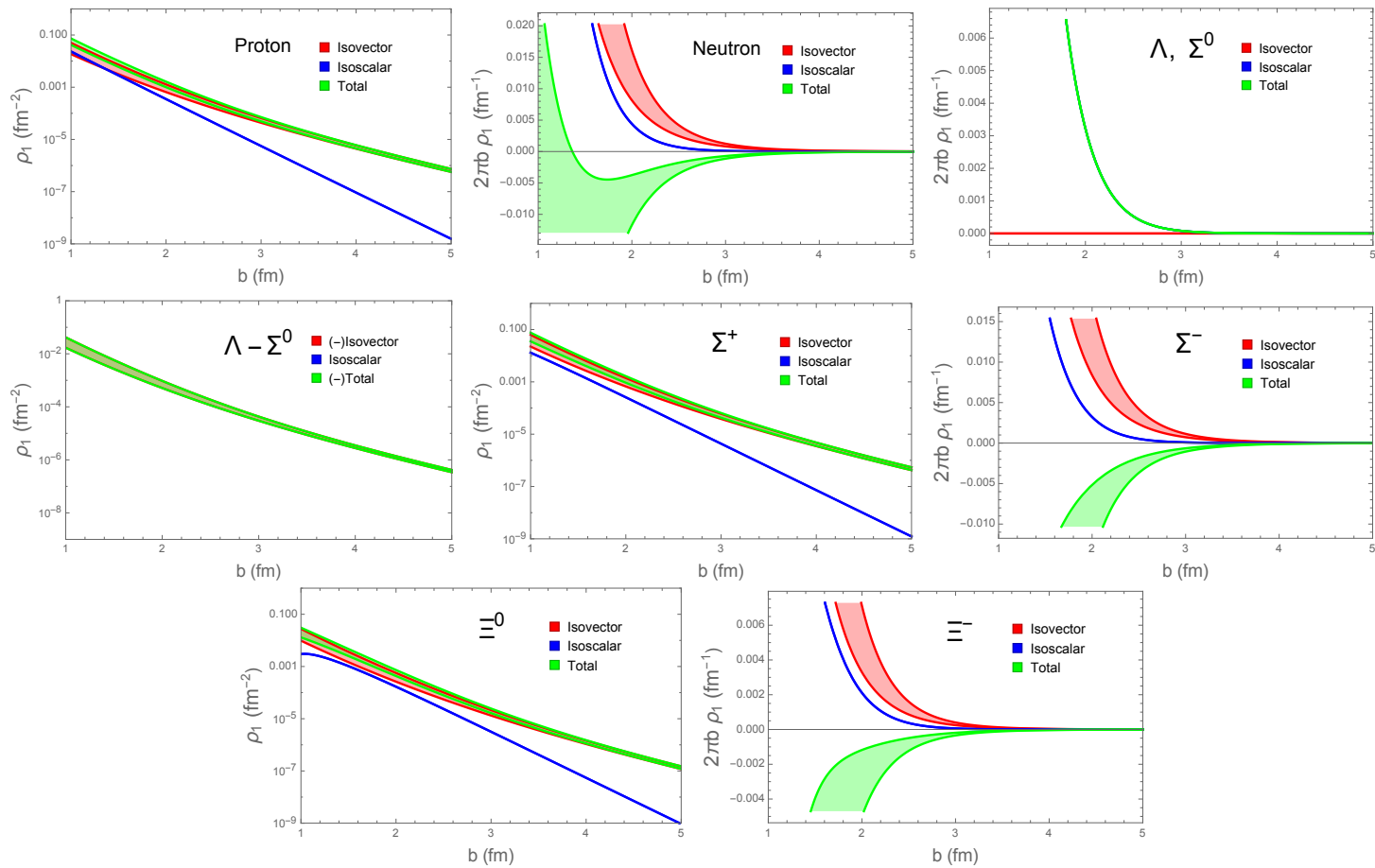

Fig. 3 Transverse charge densities of the octet baryons. Red: Isovector component calculated using $\chi \mathrm{EFT}$ and dispersive improvement. Blue: Isoscalar component estimated from vector-meson poles. Green: Total density (sum or difference of isoscalar and isovector components). For the densities with fixed sign (positive or negative) we plot $\rho_{1}(b)$ on a logarithmic scale; for those with changing sign we plot the radial densities $2 \pi b \rho_{1}(b)$ on a linear scale.

Similar features are exhibited by the magnetization densities of the octet baryons. Using the same techniques we can also calculate the flavor decomposition of the peripheral densities and study the contribution of individual quark flavors to the charge and magnetization [13.

\section{Summary and outlook}

We have presented a new approach to the calculation of the peripheral transverse densities using a combination of relativistic $\chi \mathrm{EFT}$ and dispersion analysis. The dispersive improvement extends the $\chi \mathrm{EFT}$ calculations of the isovector spectral functions into the vector-meson mass region and allows us to compute densities down to distances $\sim 1 \mathrm{fm}$ with controlled accuracy. The approach can be extended to the octet-baryon form factors of other operators (energy-momentum tensor, moments of GPDs). It can also be used to calculate the transverse densities of the decuplet baryons (especially the $\Delta$ isobar), which are studied in Lattice QCD, as well as to the densities of the octet-decuplet transition form factors (especially $N \rightarrow \Delta$ ), which are measured in resonance electroproduction.

Our approach consistently includes the contributions from decuplet intermediate states in the $\pi / K$ loop diagrams. These contributions are numerically important in the transverse densities at distances $b \sim 1-2 \mathrm{fm}$. They are also essential for ensuring the proper scaling behavior of the densities in the large $-N_{c}$ limit of QCD. This was demonstrated for the $\mathrm{SU}(2) \chi \mathrm{EFT}$ in 10,30 , and can be shown for the present $\mathrm{SU}(3)$ calculation as well.

Acknowledgements This material is based upon work supported by the U.S. Department of Energy, Office of Science, Office of Nuclear Physics under contract DE-AC05-06OR23177. This work was supported by the Spanish Ministerio de Economía y Competitividad (MINECO) and the European fund for regional development (FEDER) under contracts FIS2014-51948-C2-2-P and SEV-2014-0398. 


\section{References}

1. Brodsky SJ, Pauli HC, Pinsky SS (1998) Quantum chromodynamics and other field theories on the light cone. Phys Rept 301:299-486, DOI 10.1016/S0370-1573(97)00089-6, hep-ph/9705477

2. Soper DE (1977) The Parton Model and the Bethe-Salpeter Wave Function. Phys Rev D15:1141, DOI 10.1103/PhysRevD.15.1141

3. Burkardt M (2000) Impact parameter dependent parton distributions and off forward parton distributions for $\zeta \rightarrow 0$. Phys Rev D62:071,503, DOI 10.1103/PhysRevD.62.071503,10.1103/PhysRevD.66.119903, [Erratum: Phys. Rev.D66,119903(2002)], hep-ph/0005108

4. Miller GA (2007) Charge Density of the Neutron. Phys Rev Lett 99:112001 DOI doi:10.1103/PhysRevLett. 99.112001 arXiv:0705.2409[nucl-th]

5. Miller GA (2010) Transverse Charge Densities. Ann Rev Nucl Part Sci 60:1 DOI doi:10.1146/annurev.nucl. 012809.104508 arXiv: 1002.0355 [nucl-th]

6. Burkardt M (2003) Impact parameter space interpretation for generalized parton distributions. Int J Mod Phys A18:173 DOI doi:10.1142/S0217751X03012370 hep-ph/0207047

7. Perdrisat CF, Punjabi V, Vanderhaeghen M (2007) Nucleon Electromagnetic Form Factors. Prog Part Nucl Phys 59:694-764, DOI 10.1016/j.ppnp.2007.05.001, hep-ph/0612014

8. Punjabi V, Perdrisat CF, Jones MK, Brash EJ, Carlson CE (2015) The Structure of the Nucleon: Elastic Electromagnetic Form Factors. Eur Phys J A51:79, DOI 10.1140/epja/i2015-15079-x, 1503.01452

9. Strikman M, Weiss C (2010) Quantifying the nucleon's pion cloud with transverse charge densities. Phys Rev C82:042,201, DOI 10.1103/PhysRevC.82.042201, 1004.3535

10. Granados C, Weiss C (2014) Chiral dynamics and peripheral transverse densities. JHEP 01:092, DOI 10.1007/JHEP01(2014)092, 1308.1634

11. Granados C, Weiss C (2015) Light-tront representation of chiral dynamics in peripheral transverse densities. JHEP 1507:170 DOI doi:10.1007/JHEP07(2015)170 arXiv:1503.04839[hep-ph]

12. Granados C, Weiss C (2015) Quantum-mechanical picture of peripheral chiral dynamics. Phys Rev C92:025206 DOI doi:10.1103/PhysRevC.92.025206 arXiv: 1503.02055[hep-ph]

13. Alarcón JM, Hiller Blin AN, Meißner UG, Vicente Vacas M, Weiss C (2016) in progress

14. Miller GA, Strikman M, Weiss C (2011) Realizing vector meson dominance with transverse charge densities. Phys Rev C84:045,205, DOI 10.1103/PhysRevC.84.045205, 1105.6364

15. Belushkin MA, Hammer HW, Meißner UG (2007) Dispersion analysis of the nucleon form-factors including meson continua. Phys Rev C75:035,202, DOI 10.1103/PhysRevC.75.035202, hep-ph/0608337

16. Hemmert TR, Holstein BR, Kambor J (1997) Systematic 1/M expansion for spin 3/2 particles in baryon chiral perturbation theory. Phys Lett B395:89-95, DOI 10.1016/S0370-2693(97)00049-X, hep-ph/9606456

17. Hemmert TR, Holstein BR, Kambor J (1998) Chiral Lagrangians and delta(1232) interactions: Formalism. J Phys G24:1831-1859, DOI 10.1088/0954-3899/24/10/003, hep-ph/9712496

18. Bernard V, Fearing HW, Hemmert TR, Meissner UG (1998) The form-factors of the nucleon at small momentum transfer. Nucl Phys A635:121-145, DOI 10.1016/S0375-9474(98)00175-4,10.1016/S0375-9474(98) 00566-1, [Erratum: Nucl. Phys.A642,563(1998)], hep-ph/9801297

19. Geng LS, Martin Camalich J, Vicente Vacas MJ (2009) Leading-order decuplet contributions to the baryon magnetic moments in Chiral Perturbation Theory. Phys Lett B676:63-68, DOI 10.1016/j.physletb.2009. $04.061,0903.0779$

20. Geng LS, Martin Camalich J, Vicente Vacas MJ (2009) Electromagnetic structure of the lowest-lying decuplet resonances in covariant chiral perturbation theory. Phys Rev D80:034,027, DOI 10.1103/PhysRevD. $80.034027,0907.0631$

21. Ledwig T, Martin Camalich J, Geng LS, Vicente Vacas MJ (2014) Octet-baryon axial-vector charges and SU(3)-breaking effects in the semileptonic hyperon decays. Phys Rev D90(5):054,502, DOI 10.1103/ PhysRevD.90.054502, 1405.5456

22. Fuchs T, Gegelia J, Japaridze G, Scherer S (2003) Renormalization of relativistic baryon chiral perturbation theory and power counting. Phys Rev D68:056,005, DOI 10.1103/PhysRevD.68.056005, hep-ph/0302117

23. Frazer WR, Fulco JR (1960) Partial-Wave Dispersion Relations for the Process $\pi \pi \rightarrow N N$. Phys Rev 117:1603 DOI doi:10.1103/PhysRev.117.1603

24. Frazer WR, Fulco JR (1960) Effect of a Pion-Pion Scattering Resonance on Nucleon Structure II. Phys Rev 117:1609 DOI doi:10.1103/PhysRev.117.1609

25. Hohler G, Pietarinen E (1975) The $\rho N N$ Vertex in Vector Dominance Models. Nucl Phys B95:210 DOI doi:10.1016/0550-3213(75)90042-5

26. Hammer HW, Drechsel D, Meißner UG (2003) On the pion cloud of the nucleon. Phys Lett B586:291 DOI doi:10.1016/j.physletb.2003.12.073 hep-ph/0310240

27. Hohler G, Pietarinen E, Sabba Stefanescu 1, Borkowskı F, Simon GG, Walther VH, Wendling RD (1976) Analysis of Electromagnetic Nucleon Form-Factors. Nucl Phys B114:505-534, DOI 10.1016/0550-3213(76) 90449-1

28. Belushkin MA, Hammer HW, Meißner UG (2006) Novel evaluation of the two-pion contribution to the nucleon isovector form-factors. Phys Lett B633:507 DOI doi:10.1016/j.physletb.2005.12.053 hep-ph/0510382

29. Hoferichter M, Kubis B, Ruiz de Elvira J, Hammer HW, Meißner UG (2016) On the $\pi \pi$ continuum in the nucleon form factors and the proton radius puzzle. Eur Phys J A52:331 DOI 10.1140/epja/i2016-16331-7 arXiv: 1609.06722[hep-ph]

30. Granados C, Weiss C (2016) Light-front representation of chiral dynamics with isobar and large- $\mathrm{N}_{c}$ relations. JHEP 1606:075 DOI doi:10.1007/JHEP06(2016)075 arXiv:1603.08881 [hep-ph] 